\title{
ESTUDO DA BIOSSOLUBILIZAÇÃO DE FÓSFORO EM MEIO ÁCIDO PRODUZIDO EM REATOR AIR-LIFT
}

\author{
ANA CAROLINA F. SOLDAN ${ }^{1}$, BRUNA V. CABRAL ${ }^{1}$, TACIANA SOARES DO \\ CARMO $^{1}$, LARISSA. N. S. S. FALLEIROS ${ }^{1}$, VICELMA L. CARDOSO ${ }^{1}$ e ELOÍZIO J. \\ RIBEIRO $^{1}$
}
1 Universidade Federal de Uberlândia, Faculdade de Engenharia Química
E-mail para contato: acfs105@gmail.com.br

\begin{abstract}
RESUMO - O baixo nível de fósforo disponível no solo para as plantas é uma condição comum ao redor do mundo. Fertilizantes em grandes quantidades são utilizados a fim de suprir a deficiência desse elemento garantindo assim um melhor desenvolvimento da planta, no entanto isso gera altos custos e problemas ambientais. O presente trabalho propôs o uso de micro-organismos fúngicos excretores de ácidos orgânicos para aumentar a concentração de fósforo em solução, sendo essa uma possível alternativa, ecologicamente correta e sustentável, ao processamento químico tradicional.
\end{abstract}

\section{INTRODUÇÃO}

O fósforo possui papel importante no desenvolvimento de plantas. Ele é um dos responsáveis pela transferência de energia da célula, respiração e fotossíntese. As limitações na sua disponibilidade, no início do ciclo vegetativo, podem resultar em restrições ao desenvolvimento, das quais a planta não se recupera posteriormente, mesmo aumentando o suprimento de fósforo a níveis adequados (Grant et al., 2001).

Embora o fósforo total do solo possa ser alto, ele não está disponível para as plantas, sendo encontrado ligado firmemente a compostos orgânicos e inorgânicos. No entanto, em condições apropriadas, os minerais podem ser solubilizados e tornam-se disponíveis para as plantas e micro-organismos (Behera et. al, 2013).

O uso de fertilizantes gera altos custos e vários problemas ambientais. Como uma alternativa menos agressora ao meio ambiente e mais barata surge o termo biofertilizante definido como o uso de micro-organismos do solo para aumentar a disponibilidade e absorção de nutrientes minerais para as plantas, visando à melhoria da produtividade agrícola e tornando-se uma ferramenta promissora para a agricultura atual do mundo (Vessey, 2003). O presente trabalho visa analisar a interação dos micro-organismos na solubilização de rochas fosfatadas.

Diversos tipos de reatores têm sido utilizados para estudo do processo de biossolubiliação ganhando destaque os reatores air-lift (ARL). Os ARLs são os que melhor conferem homogeneidade de nutrientes e de iluminação ao sistema. Esses reatores consistem de duas zonas distintas, preenchidas por um líquido, na qual uma delas é de dispersão gasosa. É devido à diferença de densidade do fluido, em razão das bolhas de gás que se dispersam pela zona chamada de riser, em comparação com a zona de downcomer, que se dá a 
movimentação do fluido no reator. O principal objetivo dos ARLs é facilitar o intercâmbio de matéria entre as fases líquida e gasosa, como por exemplo, possibilitando a dissolução de $\mathrm{CO}_{2}$ da fase gasosa para a líquida, bem como a remoção de $\mathrm{O}_{2}$ produzido da fotossíntese da fase líquida para a fase gasosa (Merchunk e Gluz, 1999).

\section{MATERIAIS E MÉTODOS}

\subsection{Materiais}

Utilizou-se o concentrado fosfático (fluorapatita), com teor de 35,7\% de $\mathrm{P}_{2} \mathrm{O}_{5}$, fornecido pelo Complexo de Mineração de Tapira (Vale/Fosfértil), localizado na região do Alto Paranaíba, situado em Araxá, distante $340 \mathrm{~km}$ a oeste de Belo Horizonte, Minas Gerais. Os fungos aqui utilizados foram coletados em diferentes locais do Complexo de Mineração de Tapira (Vale/Fosfértil), localizado na região do Alto Paranaíba, Minas Gerais.

\subsection{Métodos}

Isolamento e Cultivo: $\mathrm{O}$ fungo, inicialmente, foi isolado das amostras coletadas para obtenção de culturas puras. Realizou-se o repique para a manutenção e manipulação dos isolados fúngicos. Para o isolamento e o cultivo utilizou-se placas de Petri com meio sintético seletivo sólido descrito na tabela 1 e ilustrado pela figura 1 (Pikovskaya, 1948).

Tabela 1 - Meio Sintético Seletivo Sólido (Pikovskaya, 1948).

\begin{tabular}{|c|c|}
\hline Componente & Quantidade (g/L) \\
\hline Dextrose (=D-glicose) & 10 \\
\hline Ágar & 15 \\
\hline Extrato de Levedura & 0,5 \\
\hline Fosfato Tricálcico & 5 \\
\hline Sulfato de Magnésio & 0,1 \\
\hline Sulfato de Amônio & 0,5 \\
\hline Sulfato Ferroso & 0,0001 \\
\hline Sulfato de Manganês & 0,001 \\
\hline Cloreto de Potássio & 0,2 \\
\hline
\end{tabular}

Figura 1 - Placas de Petri com meio sintético seletivo sólido

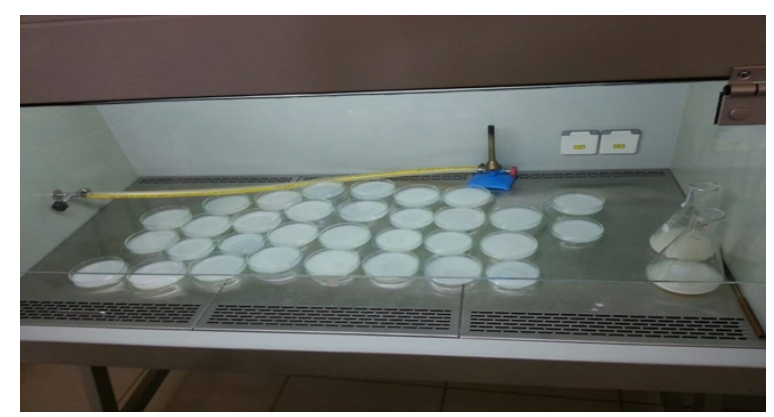


Armazenamento e preservação: As placas de Petri contendo os isolados foram armazenadas em refrigerador $\mathrm{a} \pm 4{ }^{\circ} \mathrm{C}$. Para garantir a viabilidade das culturas foram realizados cultivos periódicos por meio de repicagens de 3 a 3 meses. Utilizou-se também óleo mineral 40\% (glicerol) previamente esterilizado para cobrir totalmente as colônias contidas em eppendorfs. Para reativar o micro-organismo armazenado a $4^{\circ} \mathrm{C}$ sob óleo mineral, foi necessário repicar o mesmo para placas contendo meio sintético seletivo sólido e esperar a reativação de crescimento da cultura.

Classificação Quantitativa: Teve como objetivo identificar entre os isolados aqueles com maior capacidade de solubilização do fosfato de rocha em meio líquido. Para realização deste ensaio preparou-se o meio seletivo modificado líquido descrito na Tabela 2. Porções de $100 \mathrm{~mL}$ do meio modificado foram distribuídas em erlenmeyers de $500 \mathrm{~mL}$. Os recipientes tampados com rolhas de algodão foram esterilizados. Após resfriamento a temperatura ambiente, foram inoculadas em Erlenmeyers cerca de $10^{6}$ esporos dos isolados fúngicos selecionados sob temperatura ambiente, agitação de 100 RPM e aeração constante durante 7 dias (Sampaio et al., 2003).

Tabela 2 - Meio seletivo modificado líquido (Sampaio et al., 2003)

\begin{tabular}{|l|l|}
\hline Componente & Quantidade (g/L) \\
\hline Dextrose (= D-glicose) & 5 \\
\hline Extrato de Levedura & 0,5 \\
\hline Rocha fosfática & 5 \\
\hline Sulfato de Magnésio & 0,1 \\
\hline Sulfato de amônio & 0,5 \\
\hline Sulfato ferroso & 0,001 \\
\hline Sulfato de manganês & 0,001 \\
\hline Cloreto de potássio & 0,2 \\
\hline
\end{tabular}

Determinação do fósforo solúvel: O fósforo solúvel foi quantificado nos extratos líquidos (meio líquido) após os ensaios. A determinação do fósforo solúvel em solução se deu de acordo com o procedimento descrito em APHA - AWWA - WEF. Standard Methods for the Examination of Water and Wastewater, 20th ed. Washington, D. C.: Americam Public Health Association, 1998. - part 4000 Inorganic nonmetallic constituents, 4500-P Phosphorus.

Reator Air-lift (figura 2): Utilizou-se $3000 \mathrm{~mL}$ de meio sintético seletivo líquido (Tabela 2) exceto pela rocha fosfática em reator air-lift com aeração ascendente de ar comprimido a $0,4 \mathrm{~m} / \mathrm{s}$. Operou-se o mesmo durante 5 dias em que alíquotas eram coletadas diariamente para avaliação do crescimento celular e produção de ácidos orgânicos. O meio líquido produzido no reator air-lift após 5 dias foi empregado, em erlenmeyers, para avaliação da capacidade de biossolubilização de fósforo pelo micro-organismo 8 .

Figura 2 - Reator Air-Lift 

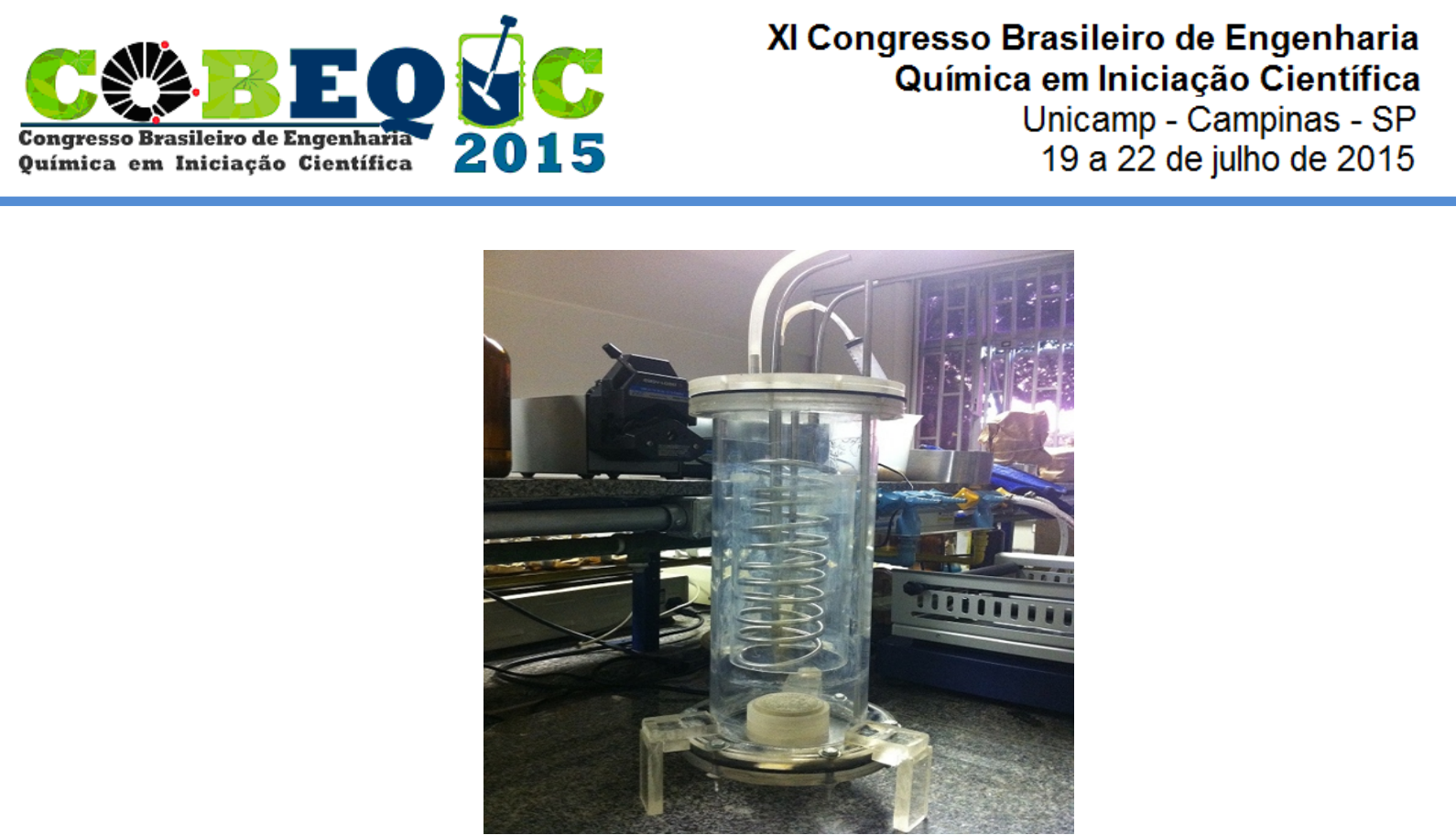

\section{RESULTADOS E DISCUSSÕES}

Através de experimentos preliminares o isolado fúngico que apresentou maior capacidade de solubilização foi o isolado 8 , ilustrado pela figura 3 , sendo o mesmo utilizado em todos experimentos seguintes.

Figura 3 - Isolado fúngico 8

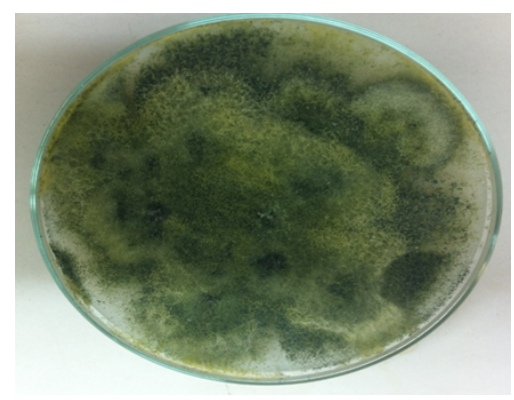

Inicialmente, em reator air-lift estudou-se o crescimento celular apresentado na Figura 4, consumo de açúcar e a produção de ácidos, os quais foram identificados e quantificados através de cromatografia líquida (HPLC), como apresentado na Tabela 3.

Figura 4 - Crescimento celular

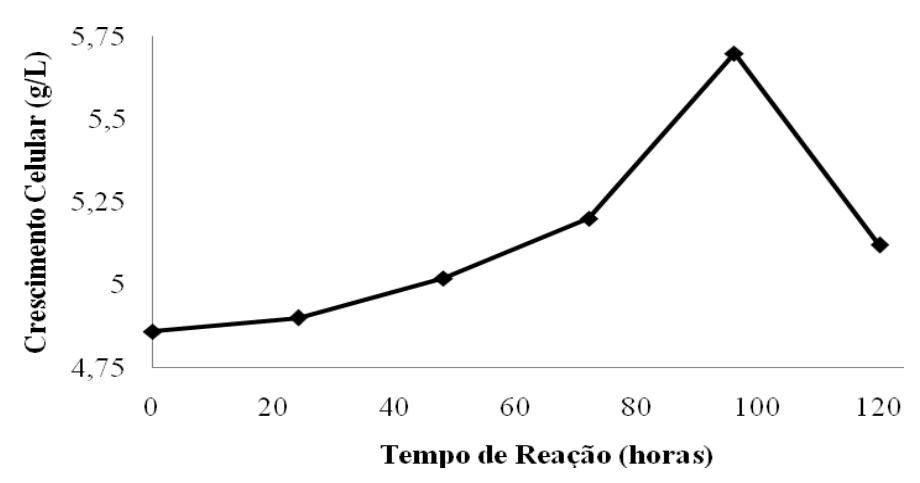


Tabela 3 - Produção de Ácidos em reator air-lift durante 5 dias empregando meio sintético seletivo líquido (Tabela 2) sem rocha com velocidade do ar comprimido igual a $0,4 \mathrm{~m} / \mathrm{s}$

\begin{tabular}{|c|c|c|c|}
\hline Dias & Ácido Lático $(\mathbf{g} / \mathbf{L})$ & Ácido Acético $(\mathbf{g} / \mathbf{L})$ & Ácido Propiônico (g/L) \\
\hline 1 & 0,0006 & 0,0117 & 0 \\
\hline 2 & 0,0015 & 0,0083 & 0,6544 \\
\hline 3 & 0,0016 & 0,0156 & 1,2710 \\
\hline 4 & 0,0262 & 0,0118 & 1,6695 \\
\hline 5 & 0,0350 & 0,0170 & 1,7359 \\
\hline
\end{tabular}

Em erlenmeyers utilizou-se um volume de $100 \mathrm{~mL}$ de meio sintético seletivo derivado do reator air-lift com adição de rocha fosfática e inóculo de micro-organismo 8 acompanhada de aeração com velocidade do ar igual a $0,4 \mathrm{~m} / \mathrm{s}$. Variou-se a concentração de rocha fosfática em 5,10 e $20 \mathrm{~g} / \mathrm{L}$ e avaliou-se a solubilização de fósforo pelo isolado fúngico 8 , como apresentado na Tabela 4. O fósforo solúvel foi quantificado através do método calorimétrico em que a curva de calibração obtida é apresentada na Equação 1:

$$
\text { Concentração de fósforo }(P P M)=34,34(\lambda)-0,4464
$$

Tabela 4 - Biossolubilização de fósforo em erlenmeyers diante de diferentes concentrações de rocha fosfática

\begin{tabular}{|c|c|c|c|}
\hline $\begin{array}{c}\text { Concentração de } \\
\operatorname{rocha}(g / L)\end{array}$ & $\begin{array}{c}\text { Tempo de } \\
\text { Reação (horas) } \\
\end{array}$ & Absorbância & $\begin{array}{c}\text { Concentração de } \\
\text { Fósforo (PPM) }\end{array}$ \\
\hline \multirow{6}{*}{ 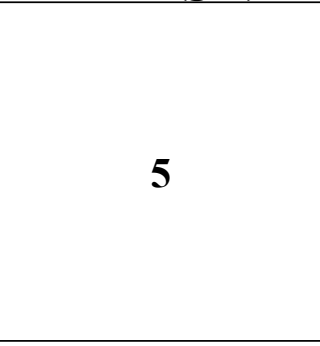 } & \multirow[b]{2}{*}{48} & 0,112 & \multirow[b]{2}{*}{40,881} \\
\hline & & 0,152 & \\
\hline & \multirow[b]{2}{*}{72} & 0,154 & \multirow[b]{2}{*}{51,166} \\
\hline & & 0,170 & \\
\hline & \multirow[b]{2}{*}{120} & 0,162 & \multirow[b]{2}{*}{85,506} \\
\hline & & 0,362 & \\
\hline \multirow{6}{*}{10} & \multirow[b]{2}{*}{48} & 0,118 & \multirow[b]{2}{*}{36,057} \\
\hline & & 0,117 & \\
\hline & \multirow[b]{2}{*}{72} & 0,151 & \multirow[b]{2}{*}{51,338} \\
\hline & & 0,174 & \\
\hline & \multirow[b]{2}{*}{120} & 0,187 & \multirow[b]{2}{*}{59,408} \\
\hline & & 0,185 & \\
\hline \multirow{6}{*}{20} & \multirow[b]{2}{*}{48} & 0,113 & \multirow[b]{2}{*}{35,713} \\
\hline & & 0,120 & \\
\hline & \multirow[b]{2}{*}{72} & 0,155 & \multirow[b]{2}{*}{46,874} \\
\hline & & 0,144 & \\
\hline & \multirow[b]{2}{*}{120} & 0,170 & \multirow[b]{2}{*}{55,287} \\
\hline & & 0,178 & \\
\hline
\end{tabular}


Ao analisar a Tabela 4 pode-se observar que houve uma maior biossolubilização de fósforo pelo isolado fúngico 8 diante de concentrações inferiores de rocha fosfática. Após 5 dias observou-se um aumento na capacidade de biossolubilização do micro-organismo empregado. Ao avaliar a condição em que empregou-se $5 \mathrm{~g} / \mathrm{L}$ de rocha fosfática nota-se que houve uma biossolubilização de $10,97 \%$ do fósforo presente na rocha fosfática, a qual apresentava inicialmente 779,24 PPM de fósforo, ou seja, 37,5\% de teor de $\mathrm{P}_{2} \mathrm{O}_{5}$.

Sabe-se do trabalho de Schneider et al. (2010) que os diferentes tipos de rocha contribuem para o $\mathrm{pH}$ e a acidez do meio e que estes parâmetros influenciam na solubilização do fósforo como pode ser visto pelos resultados por eles obtidos: rocha fosfática de Catalão Brasil com valores de $\mathrm{P}=281 \mathrm{mg} / \mathrm{L}, \mathrm{pH}=2,62 \mathrm{e}$ acidez $=3,1 \mathrm{~g} / \mathrm{L}$, a rocha de Kapuskasing Canadá obtiveram $\mathrm{P}=306 \mathrm{mg} / \mathrm{L}, \mathrm{pH}=2,57$ e acidez $=2,0 \mathrm{~g} / \mathrm{L}$ e Carolina do Norte USA valores de $\mathrm{P}=382 \mathrm{mg} / \mathrm{L}, \mathrm{pH}=3,28$ e acidez $=5,1 \mathrm{~g} / \mathrm{L}$.

Conclui-se que o uso de biofertilizantes aliados a determinados tipos de rochas são uma alternativa viável para a substituição do uso de fertilizantes químicos.

\section{REFERÊNCIAS BIBLIOGRAFICAS}

BEHERA, B. C.; SINGDEVSACHAN, S. K.; MISHRA, R.R.; DUTTA, S.K.; THATOI, H.N. Diversity, mechanism and biotechnology of phosphate solubilizing microorganism in mangrove - A review. Biocatalysis and Agricultural Biotechnology. India. p. 97-110, 2013

GRANT, C.A.; FLATEN, D.N.; TOMASIEWICZ, D.J.; SHEPPARD, S.C. A importância inicial do fósforo no desenvolvimento inicial da planta. Informações Agronômicas, Piracicaba, n. 94, p. 1-5, 2001.

MERCHUK, J. C. and Gluz M. Bioreactor, air-lift reactors. Encyclopedia of Bioprocess Technology: Fermentation, Biocatalysis and Bioseparation. Editora John Wiley \& Sons. 1999.320-324, 327, 340-344.

NEPOLEAN, P.; JAYANTHI, R.; PALLAVI,R.V. BALAMURUGAN, A.; KUBERAN,T.; BEULAH, T.; PREMKUMAR, R. Role of biofertilizers in increasing tea productivity. Asian Pacific Journal of Tropical Biomedicine. p.1443-1445, 2012.

PIKOVSKAYA, R.I. Mobilization of phosphorus in soil in connection with vital activity of some microbial species. Microbiologiya 17, 362-370, 1948.

SAMPAIO, R.M.; ALEGRE, R.M.; MARCOS, S.K.; BARROTI, G.; UBEDA, B. T. Estudo da Solubilidade de Fosfato de Rocha Tipo Tapira por Fungos do Gênero Aspergillus. In: XIV Simpósio Brasileiro de Fermentações - SINAFERM, 2003, Florianopólis. Anais do. XIV Simpósio Brasileiro de Fermentações - SINAFERM, 2003

SCHNEIDER, K.D.; VAN STRAATEN, P.; DE ORDUÑA, R.M.; GLASAUER, S.; TREVORS, J.; FALLOW, D.; SMITH, P.S. Comparing phosphorus mobilization strategies using Aspergillus niger for the mineral dissolution of three phosphate rocks. Journal of Applied Microbiology. v.108(1), p. 366-374, 2010.

VESSEY, J.K. Plant growth promoting rhizobacteria as biofertilizers. Plant and soil. Canada. n. 255, p. 571-586, 2003. 